Supplement of

\title{
Decadal changes in summertime reactive oxidized nitrogen and surface ozone over the Southeast United States
}

\section{Jingyi Li et al.}

Correspondence to: Jingqiu Mao (jmao2@alaska.edu)

The copyright of individual parts of the supplement might differ from the CC BY 4.0 License. 


\section{Supplementary Material}

Table S1. Isoprene oxidation chemistry in AM3. T represents temperature (K).

\begin{tabular}{|c|c|c|}
\hline \multirow{2}{*}{\multicolumn{3}{|c|}{$\begin{array}{r}\text { Reactions } \\
\text { Isoprene Daytime Chemistry }\end{array}$}} \\
\hline & & \\
\hline $\mathrm{ISOP}+\mathrm{OH} \rightarrow \mathrm{ISOPO} 2$ & $3.10 \times 10^{-11} \exp (350 . / \mathrm{T})$ & \\
\hline $\begin{array}{l}\mathrm{ISOPO} 2 \rightarrow 2.0 * \mathrm{HO} 2+\mathrm{CH} 2 \mathrm{O}+.333 * \mathrm{MGLY} \\
+.5 * \mathrm{GLYALD}+0.25 * \mathrm{GLYX}\end{array}$ & $4.07 \times 10^{8} \exp (-7694 . / \mathrm{T})$ & \\
\hline $\begin{array}{l}\mathrm{ISOPO} 2+\mathrm{NO} \rightarrow .90 * \mathrm{NO} 2+.90 * \mathrm{HO} 2+.9 * \mathrm{CH} 2 \mathrm{O} \\
+.55 * \mathrm{MVK}+0.35 * \mathrm{MACR}+0.1 * \mathrm{ISOPNB}\end{array}$ & $2.70 \times 10^{-12} \exp (350 . / \mathrm{T})$ & \\
\hline $\begin{array}{l}\mathrm{ISOPO} 2+\mathrm{HO} 2 \rightarrow .937 * \mathrm{ISOPOOH}+.063 * \mathrm{OH} \\
+.025 * \mathrm{MACR}+.038 * \mathrm{MVK}+.063 * \mathrm{HO} 2 \\
+.063 * \mathrm{CH} 2 \mathrm{O}\end{array}$ & $2.06 \times 10^{-13} \exp (1300 . / \mathrm{T})$ & \\
\hline $\begin{array}{l}\mathrm{ISOPO} 2+\mathrm{ISOPO} 2 \rightarrow 1.28 * \mathrm{HO} 2+.92 * \mathrm{CH} 2 \mathrm{O} \\
+.56 * \mathrm{MVK}+.36 * \mathrm{MACR}+.48 * \mathrm{ROH}+.5 * \mathrm{HC} 5\end{array}$ & $1.54 \times 10^{-13}$ & \\
\hline $\begin{array}{l}\mathrm{ISOPO} 2+\mathrm{CH} 3 \mathrm{O} 2 \rightarrow 1.1 * \mathrm{HO} 2+1.22 * \mathrm{CH} 2 \mathrm{O} \\
+.28 * \mathrm{MVK}+.18 * \mathrm{MACR}+.3 * \mathrm{HC} 5 \\
+.24 * \mathrm{CH} 3 \mathrm{OH}+.24 * \mathrm{ROH}\end{array}$ & $8.37 \times 10^{-14}$ & \\
\hline $\begin{array}{l}\mathrm{ISOPO} 2+\mathrm{CH} 3 \mathrm{CO} 3 \rightarrow .887 * \mathrm{HO} 2+.747 * \mathrm{CH} 2 \mathrm{O} \\
+.453 * \mathrm{MVK}+.294 * \mathrm{MACR}+.14 * \mathrm{HC} 5 \\
+.113 * \mathrm{DIBOO}+\{\mathrm{CO} 2\}+\mathrm{CH} 3 \mathrm{O} 2\end{array}$ & $1.68 \times 10^{-12} \exp (500 . / \mathrm{T})$ & \\
\hline $\mathrm{ISOPO} 2+\mathrm{CH} 3 \mathrm{CO} 3 \rightarrow \mathrm{MEK}+\mathrm{CH} 3 \mathrm{COOH}$ & $1.87 \times 10^{-13} \exp (500 . / \mathrm{T})$ & \\
\hline $\mathrm{ISOPNB}+\mathrm{OH} \rightarrow \mathrm{ISOPNBO} 2$ & $2.40 \times 10^{-12} \exp (745 . / \mathrm{T})$ & \\
\hline $\begin{array}{l}\text { ISOPNBO } 2+\mathrm{NO} \rightarrow .09 * \text { GLYALD }+.09 * \mathrm{HYAC} \\
+.69 * \mathrm{CH} 2 \mathrm{O}+0.88 * \mathrm{NO} 2+.44 * \mathrm{MACRN} \\
+.69 * \mathrm{HO} 2+.26 * \mathrm{MVKN}+0.21 * \mathrm{DHDN}\end{array}$ & $2.40 \times 10^{-12} \exp (360 . / \mathrm{T})$ & \\
\hline $\begin{array}{l}\mathrm{ISOPNBO} 2+\mathrm{HO} 2 \rightarrow .06 * \mathrm{GLYALD}+.06 * \mathrm{HYAC} \\
+.44 * \mathrm{CH} 2 \mathrm{O}+.28 * \mathrm{MACRN}+.16 * \mathrm{MVKN} \\
+.06 * \mathrm{NO} 2+.44 * \mathrm{HO} 2+.5 * \mathrm{OH}+.5 * \mathrm{ISNP}+ \\
0.5 * \mathrm{ROOH}\end{array}$ & $8.70 \times 10^{-14} \exp (1650 . / \mathrm{T})$ & \\
\hline $\begin{array}{l}\mathrm{ISOPNB}+\mathrm{O} 3 \rightarrow 0.05 * \mathrm{HO} 2+0.05 * \mathrm{OH}+ \\
0.11 * \mathrm{MVKN}+0.32 * \mathrm{MACRN}+0.16^{*} \mathrm{HCOOH}+ \\
0.62 * \mathrm{CH} 2 \mathrm{O}+0.36 *\{\mathrm{CO} 2\}+0.21 * \mathrm{CO}+ \\
0.06 * \mathrm{C} 4 \mathrm{NACID}+0.36 * \mathrm{HPROPN}+ \\
0.1 * \mathrm{MVKNOOH}\end{array}$ & $3.70 \times 10^{-19}$ & \\
\hline $\begin{array}{l}\mathrm{ISNP}+\mathrm{OH} \rightarrow .612 * \mathrm{OH}+.612 * \mathrm{R} 4 \mathrm{~N} 1 \\
+.386 * \mathrm{ISOPNBO} 2\end{array}$ & $4.75 \times 10^{-12} \exp (200 . / \mathrm{T})$ & \\
\hline $\begin{array}{l}\mathrm{ISOPOOH}+\mathrm{OH} \rightarrow .387 * \mathrm{ISOPO} 2+.613 * \mathrm{OH} \\
+.613 * \mathrm{HC} 5\end{array}$ & $4.75 \times 10^{-12} \exp (200 . / \mathrm{T})$ & \\
\hline $\mathrm{ISOPOOH}+\mathrm{OH} \rightarrow \mathrm{OH}+\mathrm{IEPOX}$ & $1.90 \times 10^{-11} \exp (390 . / \mathrm{T})$ & \\
\hline $\mathrm{IEPOX}+\mathrm{OH} \rightarrow \mathrm{IEPOXOO}$ & $5.78 \times 10^{-11} \exp (-400 . / \mathrm{T})$ & \\
\hline $\begin{array}{l}\mathrm{IEPOXOO}+\mathrm{HO} 2 \rightarrow .725^{*} \mathrm{HYAC} \\
+.275^{*} \mathrm{GLY} \mathrm{HLD}+.275^{*} \mathrm{GLYX}+.375^{*} \mathrm{CH} 2 \mathrm{O} \\
+.251^{*} \mathrm{CO}+.275^{*} \mathrm{MGLY}+1.125^{*} \mathrm{OH} \\
+.825^{*} \mathrm{HO} 2\end{array}$ & $2.06 \times 10^{-13} \exp (1300 . / \mathrm{T})$ & \\
\hline $\begin{array}{l}\mathrm{IEPOXOO}+\mathrm{NO} \rightarrow .725^{*} \mathrm{HYAC}+.275^{*} \mathrm{GLY} \text { ALD } \\
+.275^{*} \mathrm{GLYX}+.375^{*} \mathrm{CH} 2 \mathrm{O}+.074 * \mathrm{HCOOH}\end{array}$ & $2.70 \times 10^{-12} \exp (350 . / \mathrm{T})$ & \\
\hline
\end{tabular}




\begin{tabular}{|c|c|}
\hline \multicolumn{2}{|l|}{$\begin{array}{l}+.251 * \mathrm{CO}+\mathrm{NO} 2+.275 * \mathrm{MGLY} \\
+.125 * \mathrm{OH}+.825 * \mathrm{HO} 2\end{array}$} \\
\hline $\mathrm{HC} 5+\mathrm{OH} \rightarrow \mathrm{HC} 5 \mathrm{OO}$ & $3.35 \times 10^{-11} \exp (380 . / \mathrm{T})$ \\
\hline $\begin{array}{l}\mathrm{HC} 5+\mathrm{O} 3 \rightarrow .6 * \mathrm{MGLY}+.1 * \mathrm{OH}+.12 * \mathrm{CH} 2 \mathrm{O} \\
+.28 * \mathrm{GLYALD}+.3 * \mathrm{O} 3+.4 * \mathrm{CO}+.2 * \mathrm{H} 2 \\
+.2 * \mathrm{HYAC}+.2 * \mathrm{HCOOH}\end{array}$ & $6.16 \times 10^{-15} \exp (-1814 . / \mathrm{T})$ \\
\hline $\begin{array}{l}\mathrm{HC} 5 \mathrm{OO}+\mathrm{NO} \rightarrow \mathrm{NO} 2+.216 * \mathrm{GLYX} \\
+.234 * \mathrm{MGLY}+.234 * \mathrm{GLY} \mathrm{ALD}+.09 * \mathrm{RCHO}+ \\
\mathrm{HO} 2+.09 * \mathrm{CO}+.216 * \mathrm{HYAC}+.29 * \mathrm{DHMOB} \\
+.17 * \mathrm{MOBA}\end{array}$ & $2.35 \times 10^{-12} \exp (350 . / \mathrm{T})$ \\
\hline $\mathrm{HC} 5 \mathrm{OO}+\mathrm{NO} \rightarrow \mathrm{HNO} 3$ & $3.50 \times 10^{-13} \exp (350 . / \mathrm{T})$ \\
\hline $\begin{array}{l}\mathrm{HC} 5 \mathrm{OO}+\mathrm{HO} 2 \rightarrow .1 * \mathrm{IAP}+.9 * \mathrm{OH}+.9 * \mathrm{MGLY} \\
+.9 * \mathrm{GLYALD}+.9 * \mathrm{HO} 2\end{array}$ & $2.06 \times 10^{-13} \exp (1300 . / \mathrm{T})$ \\
\hline $\begin{array}{l}\mathrm{HC} 5 \mathrm{OO}+\mathrm{CH} 3 \mathrm{O} 2 \rightarrow .5 * \mathrm{HO} 2+.33 * \mathrm{CO}+.09 * \mathrm{H} 2 \\
+.18 * \mathrm{HY} \mathrm{AC}+.25 * \mathrm{C} 2 \mathrm{H} 5 \mathrm{OH}+.5 * \mathrm{HO} 2 \\
+.13 * \mathrm{GLYALD}+.29 * \mathrm{MGLY} \\
+.25 * \mathrm{MEK}+.95 * \mathrm{CH} 2 \mathrm{O}+.25 * \mathrm{CH} 3 \mathrm{OH}\end{array}$ & $8.37 \times 10^{-14}$ \\
\hline $\begin{array}{l}\mathrm{HC} 5 \mathrm{OO}+\mathrm{CH} 3 \mathrm{CO} 3 \rightarrow .216 * \mathrm{GLYX}+.234 * \mathrm{MGLY} \\
+.234 * \mathrm{GLYALD}+.216 * \mathrm{HYAC}+.29 * \mathrm{DHMOB} \\
+.17 * \mathrm{MOBA}+.09 * \mathrm{RCHO}+\mathrm{HO} 2+.09 * \mathrm{CO}+ \\
\mathrm{CH} 3 \mathrm{O} 2\end{array}$ & $1.68 \times 10^{-12} \exp (500 . / \mathrm{T})$ \\
\hline $\mathrm{HC} 5 \mathrm{OO}+\mathrm{CH} 3 \mathrm{CO} 3 \rightarrow \mathrm{MEK}+\mathrm{CH} 3 \mathrm{COOH}$ & $1.87 \times 10^{-13} \exp (500 . / \mathrm{T})$ \\
\hline $\begin{array}{l}\mathrm{IAP}+\mathrm{OH} \rightarrow .654 * \mathrm{OH}+.654 * \mathrm{DHMOB} \\
+.346 * \mathrm{HC} 5 \mathrm{OO}\end{array}$ & $5.31 \times 10^{-12} \exp (200 . / \mathrm{T})$ \\
\hline $\mathrm{MOBA}+\mathrm{OH} \rightarrow \mathrm{MOBAOO}$ & $2.79 \times 10^{-11} \exp (380 . / \mathrm{T})$ \\
\hline $\mathrm{MOBA}+\mathrm{O} 3 \rightarrow \mathrm{OH}+\mathrm{HO} 2+\mathrm{MEK}$ & $2.00 \times 10^{-17}$ \\
\hline $\mathrm{MOBAOO}+\mathrm{NO} \rightarrow \mathrm{RCHO}+\mathrm{HO} 2+\mathrm{NO} 2$ & $2.35 \times 10^{-12} \exp (350 . / \mathrm{T})$ \\
\hline $\mathrm{MOBAOO}+\mathrm{NO} \rightarrow \mathrm{HNO} 3$ & $3.50 \times 10^{-13} \exp (350 . / \mathrm{T})$ \\
\hline $\begin{array}{l}\mathrm{MOBAOO}+\mathrm{HO} 2 \rightarrow .5 * \mathrm{OH}+.5 * \mathrm{HO} 2 \\
+.5 * \mathrm{RCHO}+.5 * \mathrm{C} 3 \mathrm{H} 7 \mathrm{OOH}\end{array}$ & $2.06 \times 10^{-13} \exp (1300 . / \mathrm{T})$ \\
\hline $\mathrm{MVK}+\mathrm{OH} \rightarrow \mathrm{MVKO} 2$ & $2.60 \times 10^{-12} \exp (610 . / \mathrm{T})$ \\
\hline $\begin{array}{l}\mathrm{MVK}+\mathrm{O} 3 \rightarrow .202 * \mathrm{OH}+.202 * \mathrm{HO} 2 \\
+.352 * \mathrm{HCOOH}+.535 * \mathrm{CO}+.05 * \mathrm{CH} 3 \mathrm{CHO} \\
+.95 * \mathrm{MGLY}+.05 * \mathrm{CH} 2 \mathrm{O}\end{array}$ & $8.50 \times 10^{-16} \exp (-1520 . / \mathrm{T})$ \\
\hline $\begin{array}{l}\mathrm{MVKO} 2+\mathrm{NO} \rightarrow .965 * \mathrm{NO} 2+.249 * \mathrm{HO} 2 \\
+.249 * \mathrm{CH} 2 \mathrm{O}+.716^{*} \mathrm{CH} 3 \mathrm{CO} 3+.716 * \mathrm{GLY} \mathrm{ALD} \\
+.249 * \mathrm{MGLY}+.035 * \mathrm{MVKN}\end{array}$ & $2.70 \times 10^{-12} \exp (350 . / \mathrm{T})$ \\
\hline $\begin{array}{l}\mathrm{MVKO} 2+\mathrm{HO} 2 \rightarrow .38 * \mathrm{MVKOOH}+.62 * \mathrm{OH} \\
+.37 * \mathrm{GLYALD}+.37 * \mathrm{CH} 3 \mathrm{CO} 3+.13 * \mathrm{MEK} \\
+.25 * \mathrm{HO} 2+.12 * \mathrm{CH} 2 \mathrm{O}+.12 * \mathrm{MGLY}\end{array}$ & $1.82 \times 10^{-13} \exp (1300 . / \mathrm{T})$ \\
\hline $\begin{array}{l}\mathrm{MVKO} 2+\mathrm{CH} 3 \mathrm{O} 2 \rightarrow .14 * \mathrm{HO} 2+.14 * \mathrm{CH} 2 \mathrm{O} \\
+.36 * \mathrm{CH} 3 \mathrm{CO} 3+.36 * \mathrm{GLYALD}+.25 * \mathrm{ROH} \\
+.5 * \mathrm{HO} 2+.14 * \mathrm{MGLY}+.25 * \mathrm{MEK}+.75 * \mathrm{CH} 2 \mathrm{O} \\
+.25 * \mathrm{CH} 3 \mathrm{OH}\end{array}$ & $8.37 \times 10^{-14}$ \\
\hline $\begin{array}{l}\mathrm{MVKO} 2+\mathrm{CH} 3 \mathrm{CO} 3 \rightarrow .4 * \mathrm{HO} 2+.4 * \mathrm{CH} 2 \mathrm{O} \\
+.6 * \mathrm{CH} 3 \mathrm{CO} 3+.6 * \mathrm{GLYALD}+.4 * \mathrm{MGLY}+ \\
\mathrm{CH} 3 \mathrm{O} 2\end{array}$ & $1.68 \times 10^{-12} \exp (500 . / \mathrm{T})$ \\
\hline $\mathrm{MVKO} 2+\mathrm{CH} 3 \mathrm{CO} 3 \rightarrow \mathrm{MEK}+\mathrm{CH} 3 \mathrm{COOH}$ & $1.87 \times 10^{-13} \exp (500 . / \mathrm{T})$ \\
\hline
\end{tabular}




\begin{tabular}{|c|c|}
\hline $\begin{array}{l}\mathrm{MVKOOH}+\mathrm{OH} \rightarrow .791 * \mathrm{OH}+.791 * \mathrm{MEK} \\
+.209 * \mathrm{MVKO} 2\end{array}$ & $8.78 \times 10^{-12} \exp (200 . / \mathrm{T})$ \\
\hline $\begin{array}{l}\mathrm{MVKN}+\mathrm{OH} \rightarrow .65 * \mathrm{HCOOH}+\mathrm{NO} 3 \\
+.65 * \mathrm{MGLY}+.35 * \mathrm{CH} 2 \mathrm{O} \\
\end{array}$ & $1.60 \times 10^{-12}$ \\
\hline $\mathrm{MACR}+\mathrm{OH} \rightarrow .45 * \mathrm{MAO} 3+.55 * \mathrm{MACRO} 2$ & $8.00 \times 10^{-12} \exp (380 . / \mathrm{T})$ \\
\hline $\begin{array}{l}\mathrm{MACR}+\mathrm{O} 3 \rightarrow .261 * \mathrm{OH}+.202 * \mathrm{HO} 2 \\
+.326 * \mathrm{HCOOH}+.569 * \mathrm{CO}+.88 * \mathrm{MGLY}+ \\
0.12 * \mathrm{CH} 2 \mathrm{O}\end{array}$ & $1.40 \times 10^{-15} \exp (-2100 . / \mathrm{T})$ \\
\hline $\mathrm{MACR}+\mathrm{NO} 3 \rightarrow \mathrm{MAO} 3+\mathrm{HNO} 3$ & $3.40 \times 10^{-15}$ \\
\hline $\begin{array}{l}\mathrm{MACRO} 2+\mathrm{HO} 2 \rightarrow 0.42 * \mathrm{MACROOH}+0.58 * \mathrm{OH} \\
+0.58 * \mathrm{HYAC}+0.58 * \mathrm{CO}+0.58 * \mathrm{HO} 2\end{array}$ & $1.82 \times 10^{-13} \exp (1300 . / \mathrm{T})$ \\
\hline $\mathrm{MACRO} 2+\mathrm{NO} 3 \rightarrow \mathrm{NO} 2+\mathrm{HYAC}+\mathrm{CO}+\mathrm{HO} 2$ & $2.30 \times 10^{-12}$ \\
\hline $\begin{array}{l}\mathrm{MACRO} 2+\mathrm{NO} \rightarrow .97 * \mathrm{NO} 2+0.97 * \mathrm{HO} 2+ \\
0.97 * \mathrm{CO}+0.97 * \mathrm{HYAC}+.03 * \mathrm{MACRN}\end{array}$ & $2.70 \times 10^{-12} \exp (360 . / \mathrm{T})$ \\
\hline $\mathrm{MACRO} 2 \rightarrow \mathrm{CO}+\mathrm{HYAC}+\mathrm{OH}$ & 0.5 \\
\hline $\begin{array}{l}\mathrm{MACRO} 2+\mathrm{CH} 3 \mathrm{O} 2 \rightarrow .595 * \mathrm{HYAC} \\
+.255^{*} \mathrm{MGLY}+.595 * \mathrm{CO}+1.255^{*} \mathrm{CH} 2 \mathrm{O}+ \\
1.7 * \mathrm{HO} 2+.15 * \mathrm{ROH}\end{array}$ & $8.37 \times 10^{-14}$ \\
\hline $\begin{array}{l}\mathrm{MACRO} 2+\mathrm{CH} 3 \mathrm{CO} 3 \rightarrow .85 * \mathrm{HO} 2+.143 * \mathrm{MGLY} \\
+.857 * \mathrm{HYAC}+.857 * \mathrm{CO}+.143^{*} \mathrm{CH} 2 \mathrm{O}+\mathrm{CH} 3 \mathrm{O} 2 \\
\end{array}$ & $1.68 \times 10^{-12} \exp (500 . / \mathrm{T})$ \\
\hline $\mathrm{MACRO} 2+\mathrm{CH} 3 \mathrm{CO} 3 \rightarrow \mathrm{MEK}+\mathrm{CH} 3 \mathrm{COOH}$ & $1.87 \times 10^{-13} \exp (500 . / \mathrm{T})$ \\
\hline $\mathrm{MACROOH}+\mathrm{OH} \rightarrow \mathrm{MACRO} 2$ & $1.84 \times 10^{-12} \exp (200 . / \mathrm{T})$ \\
\hline $\mathrm{MACROOH}+\mathrm{OH} \rightarrow \mathrm{HYAC}+\mathrm{OH}$ & $4.40 \times 10^{-12} \exp (380 . / \mathrm{T})$ \\
\hline $\mathrm{MACRN}+\mathrm{OH} \rightarrow \mathrm{MACRNO} 2$ & $3.20 \times 10^{-12}$ \\
\hline $\begin{array}{l}\mathrm{MACRNO} 2+\mathrm{NO} \rightarrow .08 * \mathrm{CH} 3 \mathrm{COOH}+.08 * \mathrm{CH} 2 \mathrm{O} \\
+.07 * \mathrm{MGLY}+.85 * \mathrm{HY} \mathrm{AC}+1.85 * \mathrm{NO} 2+ \\
0.15 * \mathrm{NO} 3+.07 * \mathrm{HCOOH}\end{array}$ & $2.70 \times 10^{-12} \exp (350 . / \mathrm{T})$ \\
\hline $\begin{array}{l}\text { MACRNO } 2+\mathrm{HO} 2 \rightarrow .08 * \mathrm{CH} 3 \mathrm{COOH} \\
+.08 * \mathrm{CH} 2 \mathrm{O}+.15 * \mathrm{NO} 3+.07 * \mathrm{HCOOH} \\
+.07 * \mathrm{MGLY}+.85 * \mathrm{HYAC}+.85 * \mathrm{NO} 2+\mathrm{OH}\end{array}$ & $1.82 \times 10^{-13} \exp (1300 . / \mathrm{T})$ \\
\hline $\begin{array}{l}\mathrm{MAO} 3+\mathrm{NO} \rightarrow \mathrm{NO} 2+\mathrm{CH} 2 \mathrm{O}+.65 * \mathrm{CH} 3 \mathrm{O} 2+ \\
0.65 * \mathrm{CO}+.35 * \mathrm{CH} 3 \mathrm{CO} 3\end{array}$ & $8.70 \times 10^{-12} \exp (290 . / \mathrm{T})$ \\
\hline $\begin{array}{l}\mathrm{MAO} 3+\mathrm{HO} 2 \rightarrow .44 * \mathrm{OH}+.15 * \mathrm{O} 3+.44 * \mathrm{CH} 2 \mathrm{O} \\
+.29 * \mathrm{CH} 3 \mathrm{O} 2+.41 * \mathrm{MAOP}+0.15 * \mathrm{CH} 3 \mathrm{CO} 3+ \\
0.15 * \mathrm{MACO} 2 \mathrm{H}+0.29 * \mathrm{CO}\end{array}$ & $5.20 \times 10^{-13} \exp (980 . / \mathrm{T})$ \\
\hline $\begin{array}{l}\mathrm{MAO} 3+\mathrm{NO} 3 \rightarrow \mathrm{NO} 2+0.35 * \mathrm{CH} 3 \mathrm{CO} 3+\mathrm{CH} 2 \mathrm{O}+ \\
0.65^{*} \mathrm{CH} 3 \mathrm{O} 2+0.65 * \mathrm{CO}\end{array}$ & $4.00 \times 10^{-12}$ \\
\hline $\begin{array}{l}\mathrm{MAO} 3+\mathrm{CH} 3 \mathrm{O} 2 \rightarrow \mathrm{CH} 2 \mathrm{O}+\mathrm{HO} 2+\mathrm{CH} 2 \mathrm{O}+ \\
\mathrm{CH} 3 \mathrm{CO} 3\end{array}$ & $1.68 \times 10^{-12} \exp (500 . / \mathrm{T})$ \\
\hline $\mathrm{MAO} 3+\mathrm{CH} 3 \mathrm{O} 2 \rightarrow \mathrm{RCOOH}+\mathrm{CH} 2 \mathrm{O}$ & $1.87 \times 10^{-13} \exp (500 . / \mathrm{T})$ \\
\hline $\mathrm{MAO} 3+\mathrm{CH} 3 \mathrm{CO} 3 \rightarrow \mathrm{CH} 3 \mathrm{O} 2+\mathrm{CH} 2 \mathrm{O}+\mathrm{CH} 3 \mathrm{CO} 3$ & $2.50 \times 10^{-12} \exp (500 . / \mathrm{T})$ \\
\hline $\mathrm{MAO} 3+\mathrm{NO} 2+\mathrm{M} \rightarrow \mathrm{MPAN}+\mathrm{M}$ & $\begin{array}{l}\mathrm{ko}=9.00 \mathrm{E}-28 *(300 / \mathrm{T})^{8.90} \\
\mathrm{ki}=7.70 \mathrm{E}-12 *(300 / \mathrm{T})^{0.20} \\
\mathrm{f}=0.60 \\
\text { usr53 }\end{array}$ \\
\hline $\mathrm{MPAN} \rightarrow \mathrm{MAO} 3+\mathrm{NO} 2$ & $\begin{array}{l}1.111 \times 10^{28} \exp (- \\
14000 . / \mathrm{T}) * \mathrm{usr} 53\end{array}$ \\
\hline $\mathrm{MPAN}+\mathrm{OH} \rightarrow \mathrm{HYAC}+\mathrm{CO}+\mathrm{NO} 2$ & $2.90 \times 10^{-11}$ \\
\hline
\end{tabular}




\begin{tabular}{|c|c|c|}
\hline $\mathrm{MPAN}+\mathrm{O} 3 \rightarrow \mathrm{NO} 2+.6 * \mathrm{CH} 2 \mathrm{O}+\mathrm{HO} 2$ & $8.20 \times 10^{-18}$ & \\
\hline $\begin{array}{l}\mathrm{MACO} 2 \mathrm{H}+\mathrm{OH} \rightarrow 0.35 * \mathrm{CH} 3 \mathrm{CO} 3+0.65 * \mathrm{CH} 3 \mathrm{O} 2 \\
+\mathrm{CH} 2 \mathrm{O}+0.65 * \mathrm{CO}\end{array}$ & $1.51 \times 10^{-11}$ & \\
\hline $\mathrm{MAOP}+\mathrm{OH} \rightarrow \mathrm{MAO} 3$ & $6.13 \times 10^{-13} \exp (200 . / \mathrm{T})$ & \\
\hline $\mathrm{MAOP}+\mathrm{OH} \rightarrow \mathrm{MAOPO} 2$ & $3.60 \times 10^{-12} \exp (380 . / \mathrm{T})$ & \\
\hline $\mathrm{MAOPO} 2+\mathrm{HO} 2 \rightarrow \mathrm{HYAC}+2 * \mathrm{OH}$ & $1.82 \times 10^{-13} \exp (1300 . / \mathrm{T})$ & \\
\hline $\mathrm{MAOPO} 2+\mathrm{NO} \rightarrow \mathrm{HYAC}+\mathrm{OH}+\mathrm{NO} 2$ & $2.35 \times 10^{-12} \exp (350 . / \mathrm{T})$ & \\
\hline $\mathrm{MAOPO} 2+\mathrm{MAOPO} 2 \rightarrow 2 * \mathrm{HYAC}+2 * \mathrm{OH}$ & $8.37 \times 10^{-14}$ & \\
\hline $\begin{array}{l}\mathrm{MAOPO} 2+\mathrm{CH} 3 \mathrm{O} 2 \rightarrow .7 * \mathrm{HYAC}+.7 * \mathrm{OH}+ \\
\mathrm{CH} 2 \mathrm{O}+.7 * \mathrm{HO} 2+.3 * \mathrm{C} 2 \mathrm{H} 5 \mathrm{OH}\end{array}$ & $8.37 \times 10^{-14}$ & \\
\hline $\mathrm{MAOPO} 2+\mathrm{CH} 3 \mathrm{CO} 3 \rightarrow \mathrm{HYAC}+\mathrm{OH}+\mathrm{CH} 3 \mathrm{O} 2$ & $1.68 \times 10^{-12} \exp (500 . / \mathrm{T})$ & \\
\hline $\mathrm{MAOPO} 2+\mathrm{CH} 3 \mathrm{CO} 3 \rightarrow \mathrm{CH} 3 \mathrm{COOH}+\mathrm{MEK}$ & $1.87 \times 10^{-13} \exp (500 . / \mathrm{T})$ & \\
\hline $\begin{array}{l}\text { GLYALD }+\mathrm{OH} \rightarrow 0.2 * \mathrm{GLYX}+\mathrm{HO} 2+ \\
0.8 * \mathrm{CH} 2 \mathrm{O}+0.8 * \mathrm{CO}\end{array}$ & $1.00 \times 10^{-11}$ & MCM v3.3.1 \\
\hline $\mathrm{GLYX}+\mathrm{OH} \rightarrow \mathrm{HO} 2+2 * \mathrm{CO}$ & $3.10 \times 10^{-12} \exp (340 . / \mathrm{T})$ & \\
\hline $\mathrm{GLYX}+\mathrm{NO} 3 \rightarrow \mathrm{HNO} 3+\mathrm{HO} 2+2 * \mathrm{CO}$ & $\begin{array}{l}\mathrm{k}_{1}=1.4 \times 10^{-12} \exp (-1860 . / \mathrm{T}) \\
\mathrm{k}_{1} *\left(\mathrm{M}^{*} 0.21+3.5 \times 10^{18}\right) \\
/\left(\mathrm{M}^{*} 0.42+3.5 \times 10^{18}\right)\end{array}$ & \\
\hline $\mathrm{MGLY}+\mathrm{OH} \rightarrow \mathrm{CH} 3 \mathrm{CO} 3+\mathrm{CO}$ & $1.50 \times 10^{-11}$ & \\
\hline $\mathrm{MGLY}+\mathrm{NO} 3 \rightarrow \mathrm{HNO} 3+\mathrm{CO}+\mathrm{CH} 3 \mathrm{CO} 3$ & $1.40 \times 10^{-12} \exp (-1860 . / \mathrm{T})$ & \\
\hline $\mathrm{HYAC}+\mathrm{OH} \rightarrow \mathrm{MGLY}+\mathrm{HO} 2$ & $\begin{array}{l}\text { frac }=1 .-23.7 \exp (-\mathrm{T} / 70 .) ; \\
2.15 \times 10^{-12} \exp (305 . / \mathrm{T}) * \text { frac }\end{array}$ & \\
\hline $\begin{array}{l}\mathrm{HYAC}+\mathrm{OH} \rightarrow .5^{*} \mathrm{HCOOH}+\mathrm{OH} \\
+.5^{*} \mathrm{CH} 3 \mathrm{COOH}+.5^{*} \mathrm{CO}+.5^{*} \mathrm{CH} 3 \mathrm{O} 2 \\
\end{array}$ & $\begin{array}{l}2.15 \times 10^{-12} \exp (305 . / \mathrm{T}) *(1- \\
\text { frac })\end{array}$ & \\
\hline \multicolumn{3}{|l|}{ Isoprene Nighttime Chemistry } \\
\hline $\mathrm{ISOP}+\mathrm{NO} 3 \rightarrow \mathrm{INO} 2$ & $3.15 \times 10^{-12} \exp (-450 . / \mathrm{T})$ & \\
\hline $\mathrm{INO} 2+\mathrm{NO} \rightarrow \mathrm{ISN} 1+\mathrm{NO} 2+\mathrm{HO} 2$ & $2.70 \times 10^{-12} \exp (360 . / \mathrm{T})$ & MCM v3.2 \\
\hline $\mathrm{INO} 2+\mathrm{NO} 3 \rightarrow \mathrm{ISN} 1+\mathrm{NO} 2+\mathrm{HO} 2$ & $2.30 \times 10^{-12}$ & MCM v3.2 \\
\hline $\begin{array}{l}\mathrm{INO} 2+\mathrm{HO} 2 \rightarrow 0.22 * \mathrm{MVK}+0.015 * \mathrm{MACR}+ \\
0.235^{*} \mathrm{OH}+0.235 * \mathrm{NO} 2+0.235 * \mathrm{CH} 2 \mathrm{O}+ \\
0.77 * \mathrm{INPN}\end{array}$ & $2.05 \times 10^{-13} \exp (1300 . / \mathrm{T})$ & $\begin{array}{l}\text { Schwantes et } \\
\text { al.(2015) }\end{array}$ \\
\hline $\mathrm{INO} 2+\mathrm{INO} 2 \rightarrow 2.0 * \mathrm{ISN} 1+1.2 * \mathrm{HO} 2$ & $1.30 \times 10^{-12}$ & \\
\hline $\mathrm{INPN}+\mathrm{OH} \rightarrow \mathrm{ISN} 1+\mathrm{OH}$ & $1.03 \times 10^{-10}$ & \\
\hline $\mathrm{ISN} 1+\mathrm{OH} \rightarrow 0.52 * \mathrm{C} 510 \mathrm{O} 2+0.48 * \mathrm{ISNOO}$ & $4.16 \times 10^{-11}$ & \\
\hline ISN1 + NO3 $\rightarrow$ ISNOO + HNO3 & $5.95 \times 10^{-12} \exp (-1860 . / \mathrm{T})$ & \\
\hline $\begin{array}{l}\mathrm{ISN} 1+\mathrm{O} 3 \rightarrow 0.555 * \mathrm{NOA}+0.52 * \mathrm{GLYX}+ \\
0.445 * \mathrm{MGLY}+0.075 * \mathrm{H} 2 \mathrm{O} 2+0.445 * \mathrm{HO} 2+ \\
0.89 * \mathrm{CO}+0.89 * \mathrm{OH}+0.445 * \mathrm{NO} 2\end{array}$ & $2.40 \times 10^{-17}$ & \\
\hline $\begin{array}{l}\mathrm{ISNOO}+\mathrm{HO} 2 \rightarrow 0.15 * \mathrm{NC} 4 \mathrm{CO} 2 \mathrm{H}+0.15 * \mathrm{O} 3+ \\
0.41 * \mathrm{NC} 4 \mathrm{CO} 3 \mathrm{H}+0.44 * \mathrm{NOA}+0.44 * \mathrm{CO}+ \\
0.44 * \mathrm{HO} 2+0.44 * \mathrm{OH}\end{array}$ & $5.20 \times 10^{-13} \exp (980 . / \mathrm{T})$ & \\
\hline $\mathrm{ISNOO}+\mathrm{NO} \rightarrow \mathrm{NOA}+\mathrm{CO}+\mathrm{HO} 2+\mathrm{NO} 2$ & $7.50 \times 10^{-12} \exp (290 . / \mathrm{T})$ & \\
\hline ISNOO $+\mathrm{NO} 2 \rightarrow$ C5PAN1 & 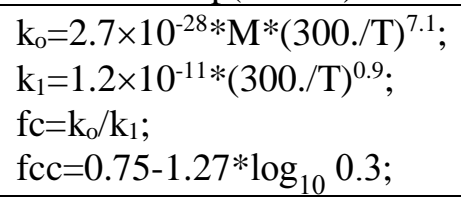 & \\
\hline
\end{tabular}




\begin{tabular}{|c|c|c|}
\hline & $\begin{array}{l}\frac{\log _{10} 0.3}{\mathrm{nfc}=10^{\left(1+\left(\log _{10} \mathrm{fc} / \mathrm{fcc}\right)^{2}\right)}} \\
\mathrm{k}_{\mathrm{o}} \mathrm{k}_{1} /\left(\mathrm{k}_{\mathrm{o}}+\mathrm{k}_{1}\right)^{*} \mathrm{nfc}\end{array}$ & \\
\hline $\mathrm{ISNOO}+\mathrm{NO} 3 \rightarrow \mathrm{NOA}+\mathrm{CO}+\mathrm{HO} 2+\mathrm{NO} 2$ & $4.00 \times 10^{-12}$ & \\
\hline $\begin{array}{l}\mathrm{ISNOO}+\mathrm{ISNOO} \rightarrow 0.6 * \mathrm{NC} 4 \mathrm{CO} 2 \mathrm{H}+1.4 * \mathrm{NOA}+ \\
1.4 * \mathrm{CO}+1.4 * \mathrm{HO} 2\end{array}$ & $1.00 \times 10^{-11}$ & \\
\hline $\mathrm{C} 510 \mathrm{O} 2+\mathrm{HO} 2 \rightarrow \mathrm{C} 510 \mathrm{OOH}$ & $2.05 \times 10^{-13} \exp (1300 . / \mathrm{T})$ & \\
\hline $\mathrm{C} 510 \mathrm{O} 2+\mathrm{NO} \rightarrow \mathrm{NO} 2+\mathrm{NOA}+\mathrm{GLYX}+\mathrm{HO} 2$ & $2.70 \times 10^{-12} \exp (360 . / \mathrm{T})$ & \\
\hline $\mathrm{C} 510 \mathrm{O} 2+\mathrm{NO} 3 \rightarrow \mathrm{NO} 2+\mathrm{NOA}+\mathrm{GLYX}+\mathrm{HO} 2$ & $2.30 \times 10^{-12}$ & \\
\hline $\begin{array}{l}\mathrm{C} 510 \mathrm{O} 2+\mathrm{C} 510 \mathrm{O} 2 \rightarrow 0.6 * \mathrm{C} 510 \mathrm{OH}+1.4 * \mathrm{NOA}+ \\
1.4 * \mathrm{GLYX}+1.4 * \mathrm{HO} 2\end{array}$ & $9.20 \times 10^{-14}$ & \\
\hline $\mathrm{C} 510 \mathrm{OH}+\mathrm{OH} \rightarrow \mathrm{NOA}+\mathrm{GLYX}+\mathrm{HO} 2$ & $2.69 \times 10^{-11}$ & \\
\hline $\mathrm{C} 510 \mathrm{OOH}+\mathrm{OH} \rightarrow \mathrm{C} 510 \mathrm{O} 2$ & $2.81 \times 10^{-11}$ & \\
\hline $\mathrm{NC} 4 \mathrm{CO} 2 \mathrm{H}+\mathrm{OH} \rightarrow \mathrm{NOA}+\mathrm{HO} 2+\mathrm{CO}$ & $2.16 \times 10^{-11}$ & \\
\hline $\mathrm{NC} 4 \mathrm{CO} 3 \mathrm{H}+\mathrm{OH} \rightarrow \mathrm{ISNOO}$ & $2.52 \times 10^{-11}$ & \\
\hline $\mathrm{NOA}+\mathrm{OH} \rightarrow \mathrm{MGLY}+\mathrm{NO} 2$ & $1.00 \times 10^{-12}$ & \\
\hline $\mathrm{C} 5 \mathrm{PAN} 1 \rightarrow \mathrm{ISNOO}+\mathrm{NO} 2$ & 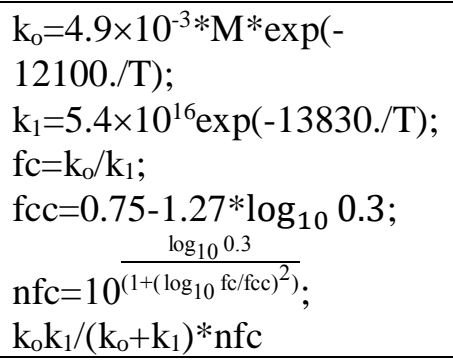 & \\
\hline $\mathrm{C} 5 \mathrm{PAN} 1+\mathrm{OH} \rightarrow \mathrm{NOA}+\mathrm{CO}+\mathrm{CO}+\mathrm{NO} 2$ & $2.16 \times 10^{-11}$ & \\
\hline \multicolumn{3}{|l|}{ Isoprene Ozonolysis } \\
\hline $\begin{array}{l}\mathrm{ISOP}+\mathrm{O} 3 \rightarrow .325 * \mathrm{MACR}+.244 * \mathrm{MVK} \\
+.845 * \mathrm{CH} 2 \mathrm{O}+.11 * \mathrm{H} 2 \mathrm{O} 2+.27 * \mathrm{OH}+.128 * \mathrm{C} 3 \mathrm{H} 6 \\
+.051 * \mathrm{CH} 3 \mathrm{O} 2+.522 * \mathrm{CO}+.204 * \mathrm{HCOOH} \\
+.199 * \mathrm{CH} 3 \mathrm{CO} 3+.026 * \mathrm{HO} 2\end{array}$ & $1.00 \times 10^{-14} \exp (-1970 . / \mathrm{T})$ & \\
\hline \multicolumn{3}{|l|}{ Photolysis of major organic nitrates } \\
\hline $\mathrm{ISOPNB}+\mathrm{h} v \rightarrow \mathrm{HC} 5+\mathrm{NO} 2+\mathrm{HO} 2$ & $1.21 \times 10^{-6} \mathrm{~s}^{-1}$ & $\begin{array}{l}\text { Calculated } \\
\text { based on } 24-\mathrm{hr} \\
\text { average of } \\
\text { modeled results }\end{array}$ \\
\hline $\mathrm{MACRN}+\mathrm{h} v \rightarrow \mathrm{NO} 2+\mathrm{HYAC}+\mathrm{HO} 2+\mathrm{CO}$ & $8.48 \times 10^{-5} \mathrm{~s}^{-1}$ & \\
\hline $\mathrm{MVKN}+\mathrm{h} v \rightarrow \mathrm{GLYALD}+\mathrm{NO} 2+\mathrm{CH} 3 \mathrm{CO} 3$ & $1.36 \times 10^{-5} \mathrm{~s}^{-1}$ & \\
\hline $\mathrm{NOA}+\mathrm{h} v \rightarrow \mathrm{CH} 3 \mathrm{CO} 3+\mathrm{CH} 2 \mathrm{O}+\mathrm{NO} 2$ & $8.48 \times 10^{-6} \mathrm{~s}^{-1}$ & \\
\hline $\mathrm{ISN} 1+\mathrm{h} v \rightarrow \mathrm{NOA}+2.0 * \mathrm{CO}+2.0 * \mathrm{HO} 2$ & $1.36 \times 10^{-4} \mathrm{~s}^{-1}$ & \\
\hline TERPN1 + h $v \rightarrow$ NO2 & $1.21 \times 10^{-6} \mathrm{~s}^{-1}$ & \\
\hline TERPN2 + h $v \rightarrow$ NO2 & $1.21 \times 10^{-6} \mathrm{~s}^{-1}$ & \\
\hline
\end{tabular}


Table S2. Monoterpene oxidation chemistry in AM3. T represents temperature $(\mathrm{K})$.

\begin{tabular}{|l|l|l|}
\hline \multicolumn{1}{|c|}{ Reactions } & \multicolumn{1}{c|}{$\begin{array}{c}\text { Reaction Rates } \\
\left(\mathrm{molecule}^{-1} \mathrm{~cm}^{3} \mathrm{~s}^{-1}\right)\end{array}$} & \multicolumn{1}{|c|}{ Note } \\
\hline $\mathrm{C} 10 \mathrm{H} 16+\mathrm{OH} \rightarrow \mathrm{TERPO} 2$ & $1.2 \times 10^{-11} \mathrm{exp}(440 / \mathrm{T})$ & $\begin{array}{l}\text { Atkinson and } \\
\text { Arey (2003) }\end{array}$ \\
\hline $\mathrm{TERPO} 2+\mathrm{NO} \rightarrow 0.74 * \mathrm{NO} 2+0.26 * \mathrm{TERPN} 1$ & $2.7 \times 10^{-12} \exp (360 / \mathrm{T})$ & $\begin{array}{l}\text { Saunders et al. } \\
(2003)\end{array}$ \\
\hline TERPO2 + HO2 $\rightarrow$ products & $2.9 \times 10^{-13} \exp (1300 / \mathrm{T})$ & $\begin{array}{l}\text { Saunders et al. } \\
(2003)\end{array}$ \\
\hline TERPO2 + TERPO2 $\rightarrow$ products & $1.68 \times 10^{-12} \exp (500 / \mathrm{T})$ & $\begin{array}{l}\text { Tyndall et al. } \\
(2001)\end{array}$ \\
\hline $\mathrm{C} 10 \mathrm{H} 16+\mathrm{O} 3 \rightarrow$ products & $5.30 \times 10^{-16} \mathrm{exp}(-530 / \mathrm{T})$ & RCAM2 \\
\hline $\mathrm{C} 10 \mathrm{H} 16+\mathrm{NO} 3 \rightarrow 0.1 * \mathrm{TERPN} 2+0.9 * \mathrm{NO} 2$ & $1.2 \times 10^{-12} \exp (490 / \mathrm{T})$ & $\begin{array}{l}\text { Atkinson and } \\
\text { Arey }(2003)\end{array}$ \\
\hline
\end{tabular}


Table S3. Heterogeneous loss of related species in the model.

\begin{tabular}{|l|l|l|}
\hline Reactions & $\begin{array}{l}\text { Reaction Rate or Uptake } \\
\text { Coefficient }\end{array}$ & Note \\
\hline GLYX $\rightarrow$ AGLYX & $\gamma_{\mathrm{glyx}}=1.0 \times 10^{-3}$ & \\
\hline MGLY $\rightarrow$ AMGLY & $\gamma_{\mathrm{mgly}}=1.0 \times 10^{-7}$ & \\
\hline IEPOX $\rightarrow$ AIEPOX & $\gamma_{\text {iepox }}=1.0 \times 10^{-3}$ & $\begin{array}{l}\text { Fisher et } \\
\text { al.(2016) }\end{array}$ \\
\hline ISOPNB $\rightarrow$ AONJ & $\gamma_{\mathrm{IN}}=5.0 \times 10^{-3}$ & $\begin{array}{l}\text { Fisher et } \\
\text { al.(2016) }\end{array}$ \\
\hline TERPN1 $\rightarrow$ AONJ & $\gamma_{\mathrm{MN}}=1.0 \times 10^{-2}$ & $\begin{array}{l}\text { Pye et } \\
\text { al.(2015) }\end{array}$ \\
\hline AONJ $\rightarrow$ HNO3 + ROH & $\mathrm{k}=9.26 \times 10^{-5} \mathrm{~s}^{-1}$ & \\
\hline
\end{tabular}


Table S4. Statistical analysis of ozone and major RON species from the base case and no_hydro case ${ }^{\mathrm{a}}$.

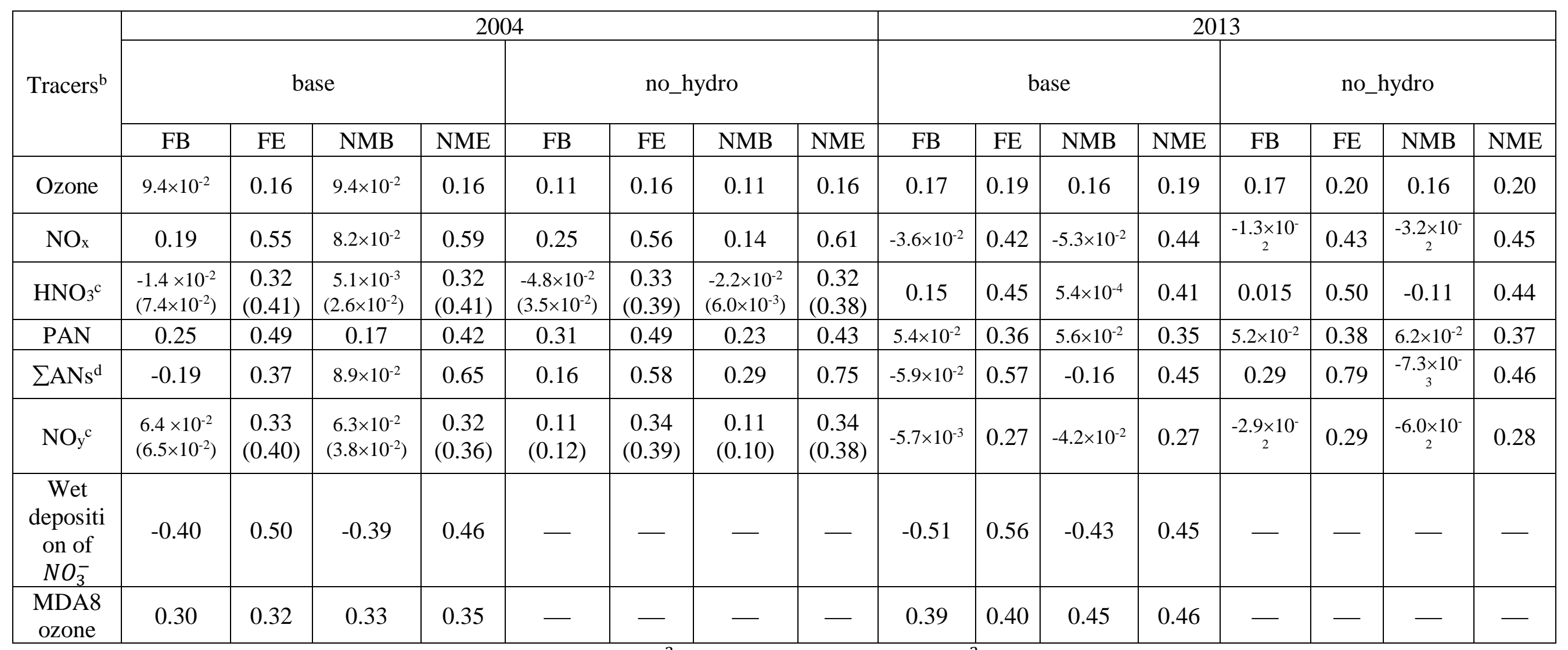

aDescriptions of the two cases can be found in Table 2. $\mathrm{FB}=\frac{2}{N} \sum_{i=1}^{N}\left(P_{i}-O_{i}\right) /\left(P_{i}+O_{i}\right), \mathrm{FE}=\frac{2}{N} \sum_{i=1}^{N}\left|P_{i}-O_{i}\right| /\left(P_{i}+O_{i}\right)$,

$\mathrm{NMB}=\sum_{i=1}^{N}\left(P_{i}-O_{i}\right) / \sum_{i=1}^{N} O_{i}, \mathrm{MNE}=\frac{1}{N} \sum_{i=1}^{N}\left|P_{i}-O_{i}\right| / \sum_{i=1}^{N} O_{i}$, where $\mathrm{P}_{\mathrm{i}}$ and $\mathrm{O}_{\mathrm{i}}$ are modeled and observed data, and $\mathrm{N}$ is number of valid data. 
${ }^{b}$ For ozone, $\mathrm{NO}_{\mathrm{x}}, \mathrm{HNO}_{3}$, PAN, $\sum$ ANs and $\mathrm{NO}_{\mathrm{y}}$, observations are from ICARTT, SENEX and SEAC ${ }^{4} \mathrm{RS}$ within the boundary layer $(<1.5 \mathrm{~km})$; for wet deposition of $\mathrm{NO}_{3}^{-}$, observations are from NADP; for MDA8 ozone, observations are from EPA AQS data during July-August of 2004 and 2013 at monitoring stations in Fig. S2.

'Statistical results of $\mathrm{HNO}_{3}$ and $\mathrm{NO}_{\mathrm{y}}$ in 2004 (ICARTT) outside of and within the brackets used observed $\mathrm{HNO}_{3}$ measured by mist chamber/IC by University of New Hampshire and Chemical Ionization Mass Spectrometer (CIMS) by California Institute of Technology, respectively.

${ }^{\mathrm{d}}$ Statistical results of $\sum$ ANs in 2013 used observations during SEAC ${ }^{4} \mathrm{RS}$. 
Table S5. Lifetimes of major organic nitrates from isoprene and monoterpenes in AM3.

\begin{tabular}{|c|c|c|}
\hline Species in AM3 & Formation Pathways & Lifetime $^{a}$ \\
\hline ISOPNB $^{b}$ & $\begin{array}{l}\mathrm{C}_{5} \beta \text {-hydroxy nitrate from } \\
\text { isoprene oxidation by } \mathrm{OH}\end{array}$ & $1.2(1.4)$ \\
\hline ISN1 & $\begin{array}{l}\mathrm{C}_{5} \text { carbonyl nitrate from } \\
\text { isoprene oxidation by } \mathrm{NO}_{3}\end{array}$ & $3.6(3.5)$ \\
\hline INPN & $\begin{array}{l}\mathrm{C}_{5} \text { nitrooxy hydroperoxide } \\
\text { from isoprene oxidation by } \\
\mathrm{NO}_{3}\end{array}$ & $8.5(7.8)$ \\
\hline MVKN & $\begin{array}{c}\text { Methyl vinyl ketone nitrate } \\
\text { from ISOPNB oxidation } \\
\text { by OH }\end{array}$ & $9.6(9.3)$ \\
\hline MACRN & $\begin{array}{l}\text { Methacrolein nitrate from } \\
\text { ISOPNB oxidation by } \mathrm{OH}\end{array}$ & $1.5(1.4)$ \\
\hline ISNP & $\begin{array}{l}\mathrm{C}_{5} \text { nitrooxy hydroperoxide } \\
\text { from isoprene oxidation by } \\
\mathrm{OH}\end{array}$ & $12(12)$ \\
\hline PROPNN & $\begin{array}{c}\text { Propanone nitrate from } \\
\text { oxidation of INPN and } \\
\text { ISN1 } \\
\end{array}$ & $17.6(16.2)$ \\
\hline $\mathrm{DHDN}^{\mathrm{c}}$ & $\begin{array}{c}\mathrm{C}_{5} \text { dihydroxy dinitrate } \\
\text { from ISOPNB oxidation } \\
\text { by } \mathrm{OH}\end{array}$ & $11.4(10.3)$ \\
\hline TERPN1 & $\begin{array}{c}\text { Nitrate from monoterpene } \\
\text { oxidation by } \mathrm{OH}\end{array}$ & $19.6(18.8)$ \\
\hline TERPN2 & $\begin{array}{l}\text { Nitrate from monoterpene } \\
\text { oxidation by } \mathrm{NO}_{3}\end{array}$ & $14.1(14.4)$ \\
\hline PAN & & $0.72(0.66)$ \\
\hline $\mathrm{HNO}_{3}$ & & $16(16)$ \\
\hline
\end{tabular}

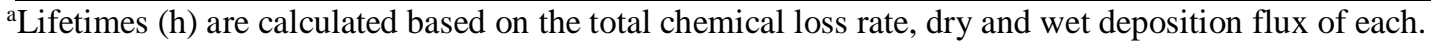
compound during July-August of 2004; values in brackets are from July-August of 2013. It should be noted that the lifetimes listed here are 24-h average, different from estimates by Müller et al. (2014).

${ }^{b}$ The lifetime of ISOPNB includes impacts of aerosol hydrolysis.

${ }^{\mathrm{c}}$ The lifetime of DHDN is based on dry and wet deposition since it is not removed by chemical oxidation. 
Table S6. Non-radical species used in AM3.

\begin{tabular}{|c|c|}
\hline Species Name & Description \\
\hline ISOP & isoprene \\
\hline $\mathrm{CH} 2 \mathrm{O}$ & formaldehyde \\
\hline MGLY & methyl-glyoxal \\
\hline GLYALD & glycolaldehyde \\
\hline GLYX & glyoxal \\
\hline MVK & methylvinyl ketone \\
\hline MACR & methacrolein \\
\hline ISOPNB & see Table S5 \\
\hline ISOPOOH & hydroxy hydroperoxide from isoprene oxidation by $\mathrm{OH}$ \\
\hline HC5 & $\mathrm{C}_{5} \mathrm{H}_{8} \mathrm{O}_{2}$, see Paulot et al. (2009) \\
\hline $\mathrm{ROH}$ & alcohol with the carbon number $\geq 3$ \\
\hline $\mathrm{CH} 3 \mathrm{OH}$ & methanol \\
\hline MEK & ketones with the carbon number $>3$ \\
\hline $\mathrm{CH} 3 \mathrm{COOH}$ & methyl hydroperoxide \\
\hline HYAC & hydroxyacetone \\
\hline MACRN & see Table S5 \\
\hline MVKN & see Table S5 \\
\hline DHDN & see Table S5 \\
\hline ISNP & see Table S5 \\
\hline $\mathrm{ROOH}$ & hydroperoxides with the carbon number $\geq 3$ \\
\hline $\mathrm{HCOOH}$ & formic acid \\
\hline C4NACID & $\mathrm{C}_{4}$ hydroxy nitrooxy carboxylic acid, see Lee et al. (2014) \\
\hline HPROPN & hydroxy propyl nitrate, see Lee et al. (2014) \\
\hline MVKNOOH & MVKN with one additional hydroperoxide group, see Lee et al. (2014) \\
\hline IEPOX & isoprene epoxydiol \\
\hline RCHO & propyl aldehyde \\
\hline DHMOB & $\mathrm{C}_{5} \mathrm{H}_{8} \mathrm{O}_{4}$, see Paulot et al. (2009) \\
\hline MOBA & C5 acid from isoprene \\
\hline IAP & peroxides from $\mathrm{HC} 5$ oxidation by $\mathrm{OH}$ \\
\hline $\mathrm{C} 3 \mathrm{H} 7 \mathrm{OOH}$ & propyl hydroperoxide \\
\hline $\mathrm{CH} 3 \mathrm{CHO}$ & acetaldehyde \\
\hline MACROOH & hydroperoxide from MACR oxidation by $\mathrm{OH}$ \\
\hline MAOP & peroxide from MAO3 \\
\hline MACO2H & organic peroxide \\
\hline MPAN & peroxymethacryloyl nitrate \\
\hline $\mathrm{C} 2 \mathrm{H} 5 \mathrm{OH}$ & alcohol \\
\hline ISN1 & see Table S5 \\
\hline INPN & see Table S5 \\
\hline NOA & propanone nitrate \\
\hline $\mathrm{NC} 4 \mathrm{CO} 2 \mathrm{H}$ & $\begin{array}{l}\mathrm{C}_{5} \text { nitrate with a carboxylic acid group from isoprene oxidation by } \\
\mathrm{NO}_{3} \text {, see } \mathrm{MCM} \vee 3.2\end{array}$ \\
\hline
\end{tabular}




\begin{tabular}{|l|l|}
\hline $\mathrm{NC} 4 \mathrm{CO} 3 \mathrm{H}$ & $\begin{array}{l}\mathrm{C}_{5} \text { nitrate with a peroxy acid group from isoprene oxidation by } \mathrm{NO}_{3}, \\
\text { see } \mathrm{MCM} \text { v3.2 }\end{array}$ \\
\hline C5PAN1 & $\mathrm{C}_{5}$ peroxy nitrate from isoprene oxidation by $\mathrm{NO}_{3}$, see MCM v3.2 \\
\hline C510OOH & $\begin{array}{l}\mathrm{C}_{5} \text { nitrate with a hydroperoxide group from isoprene oxidation by } \\
\mathrm{NO}_{3} \text {, see } \mathrm{MCM} \text { v3.2 }\end{array}$ \\
\hline C510OH & $\mathrm{C}_{5}$ nitrate from isoprene oxidation by $\mathrm{NO}_{3}$, see MCM v3.2 \\
\hline C3H6 & propylene \\
\hline C10H16 & monoterpenes \\
\hline TERPN1 & see Table S5 \\
\hline TERPN2 & see Table S5 \\
\hline
\end{tabular}



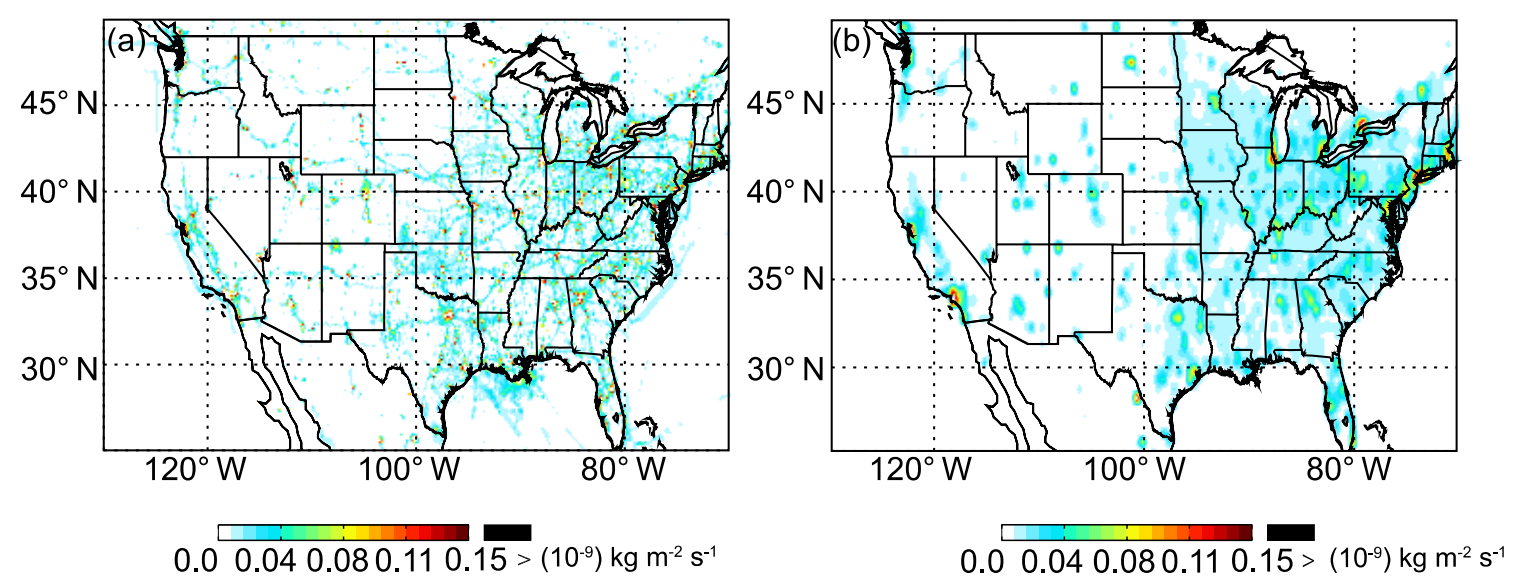

Figure S1. Anthropogenic $\mathrm{NO}_{x}$ emission rate during July-August 2013 of (a) NEI11v1 inventory and (b) RCP8.5. 


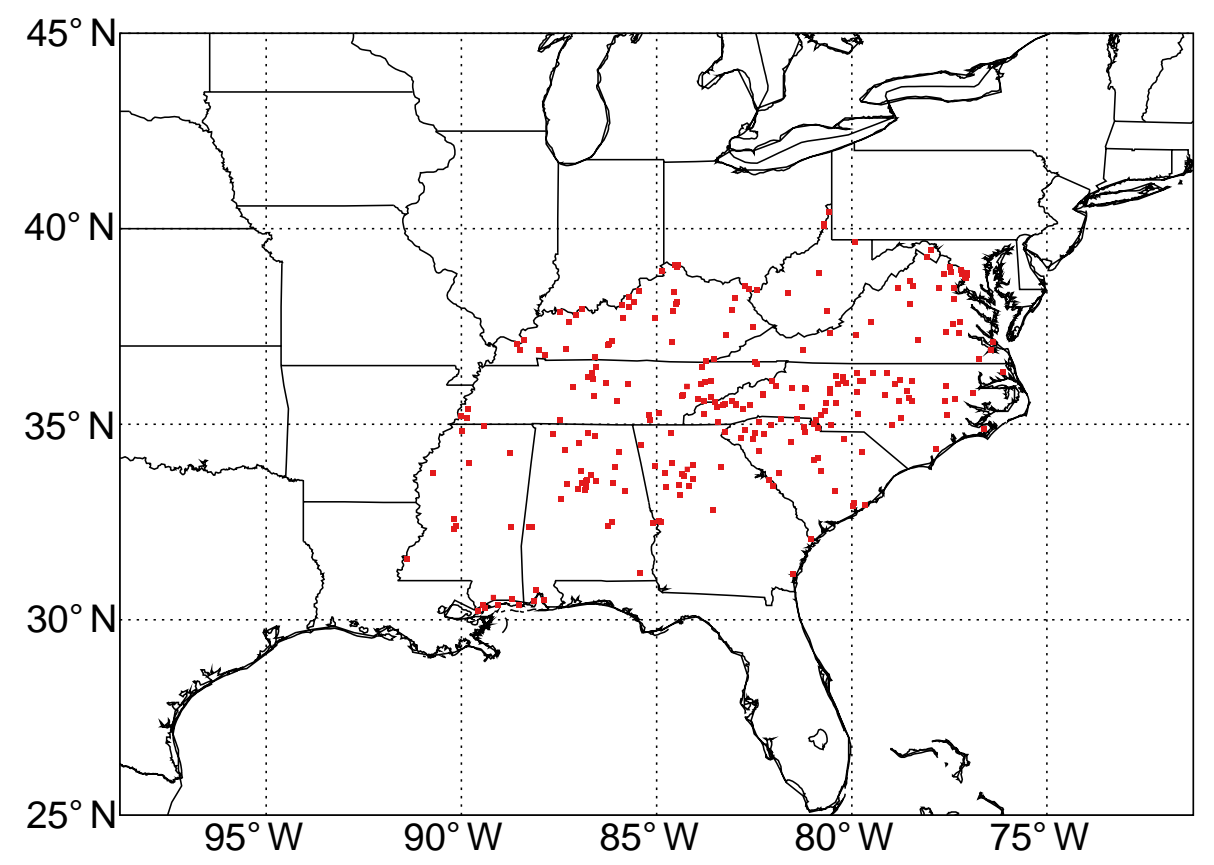

Figure S2. EPA AQS ozone monitoring sites in the Southeast U.S., from which EPA provides the MDA8 metric used in our study. 

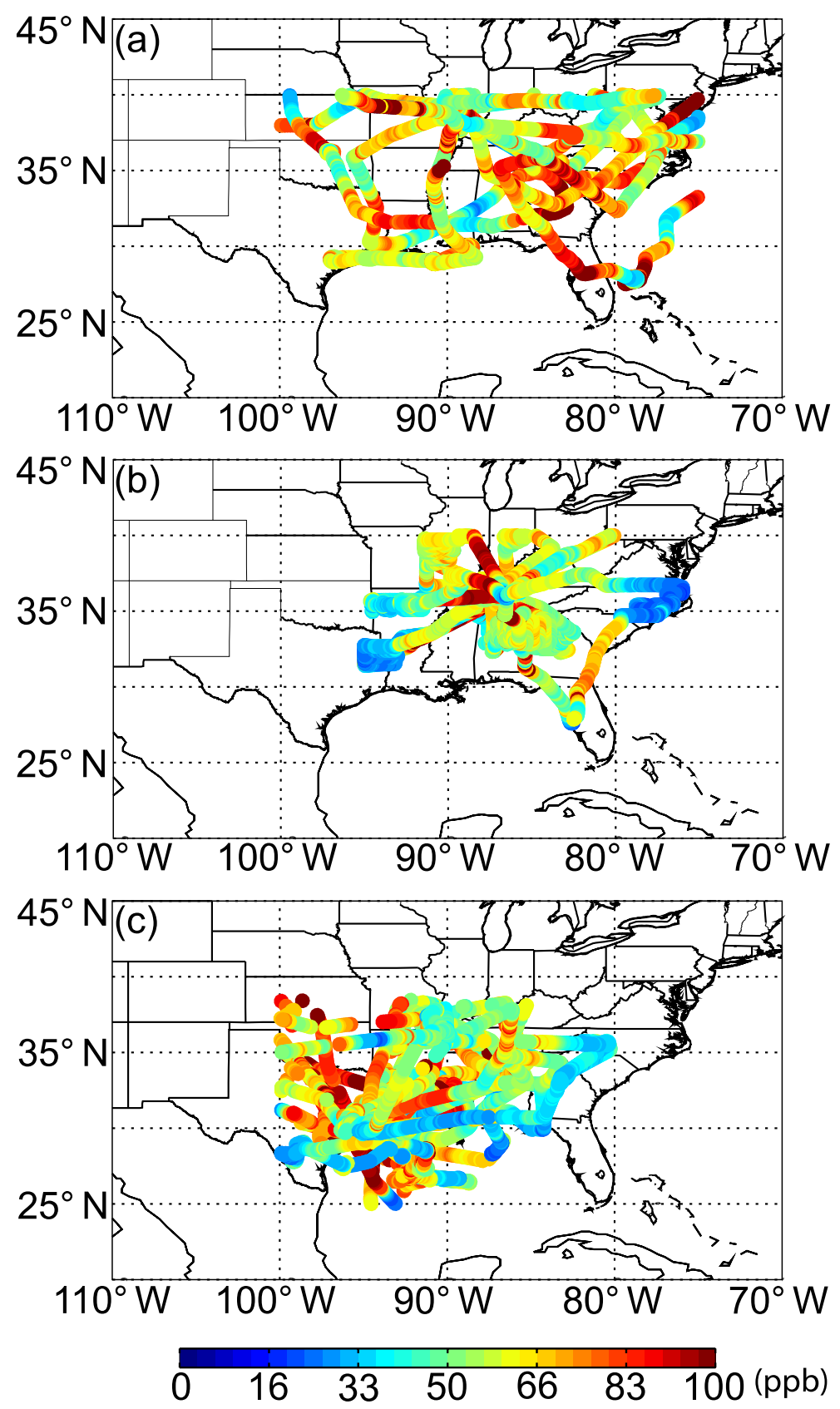

Figure S3. Ozone (ppb) along daytime flight tracks during (a) ICARTT (up to $12 \mathrm{~km}$ ) (b) SENEX (up to $6 \mathrm{~km}$ ) and (c) SEAC ${ }^{4} \mathrm{RS}$ (up to $12.5 \mathrm{~km}$ ). Data from biomass burning, urban plumes, stratospheric air and outside of the $25-40^{\circ} \mathrm{N}$ latitude and $100-75^{\circ} \mathrm{W}$ longitude region are excluded. 


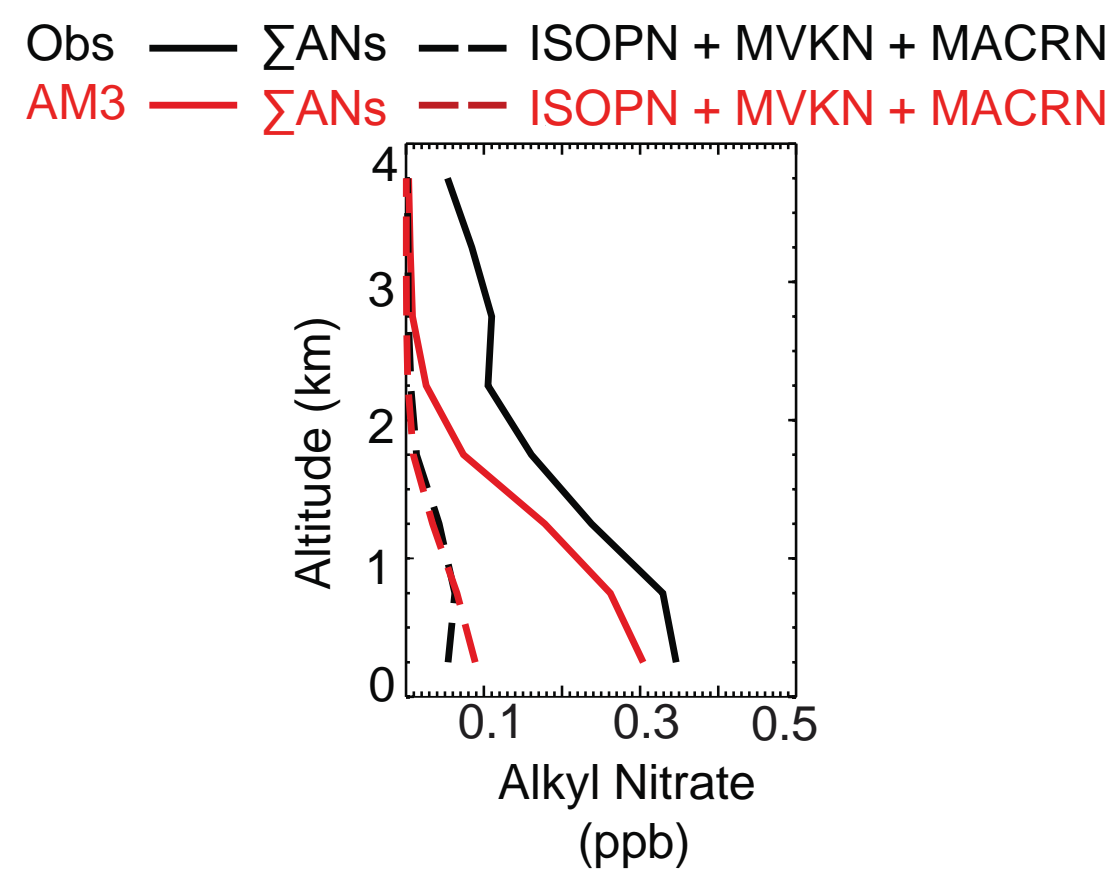

Figure S4. Mean vertical profiles of $\sum$ ANs (solid lines) and sum of ISOPN, MVKN and MACRN (dashed lines) during SEAC ${ }^{4} \mathrm{RS}$ from observations (black) and AM3 with hydrolysis of ISOPNB (red). The discrepancy between $\sum$ ANs and sum of ISOPN, MVKN and MACRN is attributed to monoterpene nitrates and a C5 dihydroxy dinitrate (DHDN) and nighttime $\mathrm{NO}_{3}$ oxidation products from isoprene. 

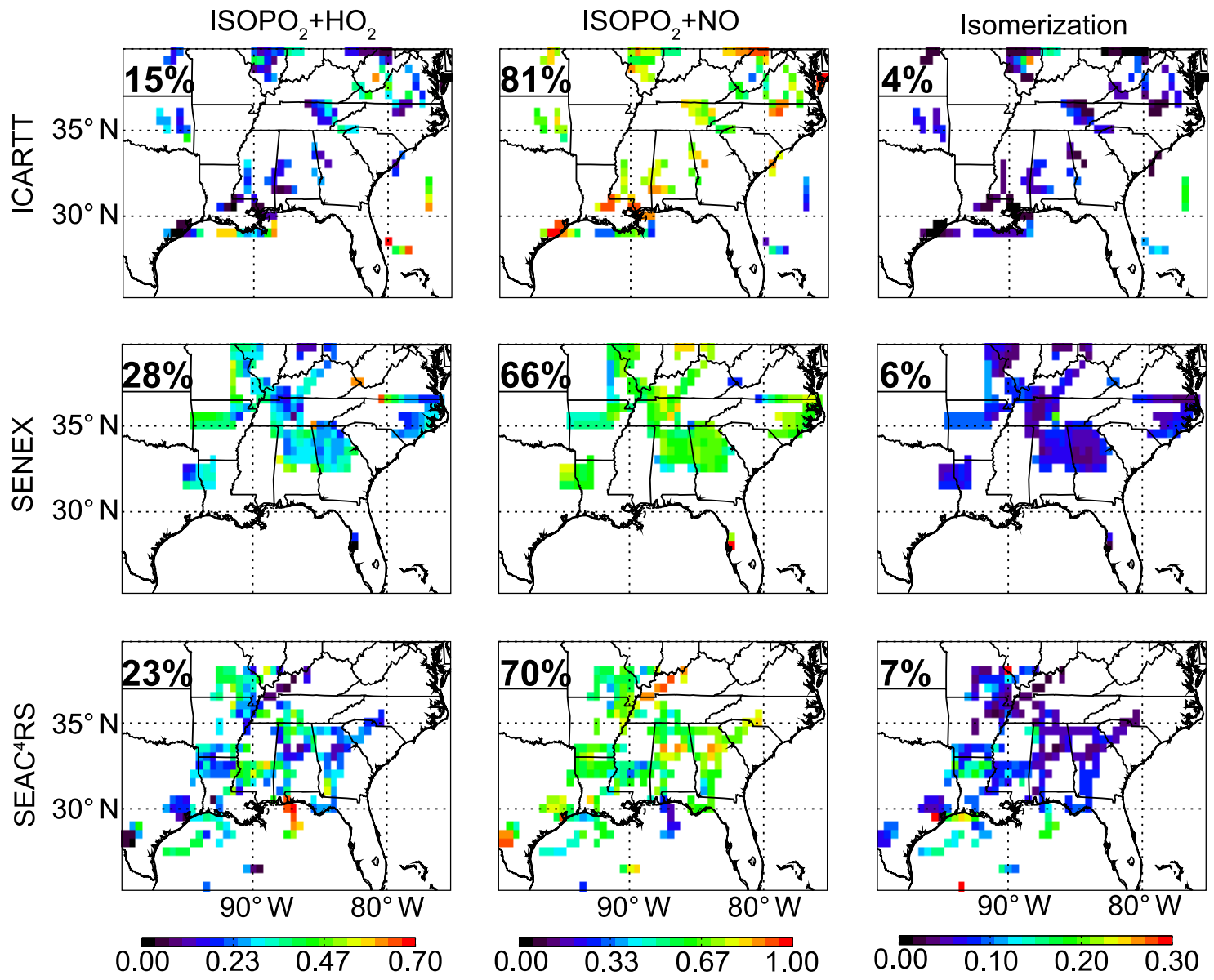

Figure S5. Ratio of major $\mathrm{ISOPO}_{2}$ loss pathways to the total $\mathrm{ISOPO}_{2}$ loss (sum of the three individual pathways) during ICARTT (July-August of 2004, top), SENEX (June-July of 2013, middle) and SEAC ${ }^{4}$ RS (July-September of 2013, bottom) in the boundary layer (below $1.5 \mathrm{~km}$ ). Bold number in each panel is the mean percentage of each pathway to the total $\mathrm{ISOPO}_{2}$ loss. 

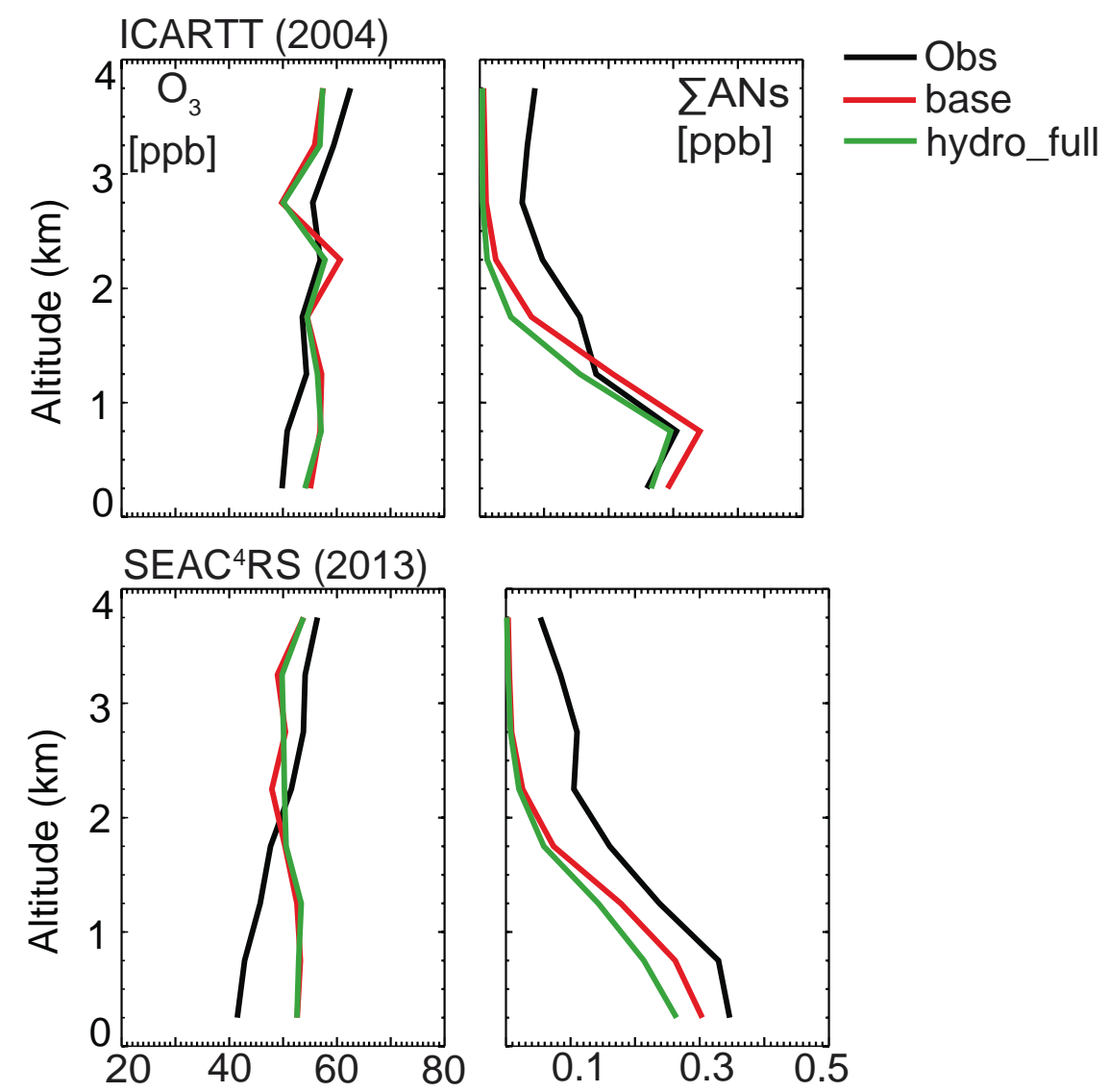

Figure S6. Mean vertical profiles of ozone and $\sum$ ANs during ICARTT (top) and $\mathrm{SEAC}^{4} \mathrm{RS}$ (bottom). Red lines are results of AM3 with ISOPNB hydrolysis only; green lines are from AM3 with hydrolysis of ISOPNB, DHDN and TERPN1. 


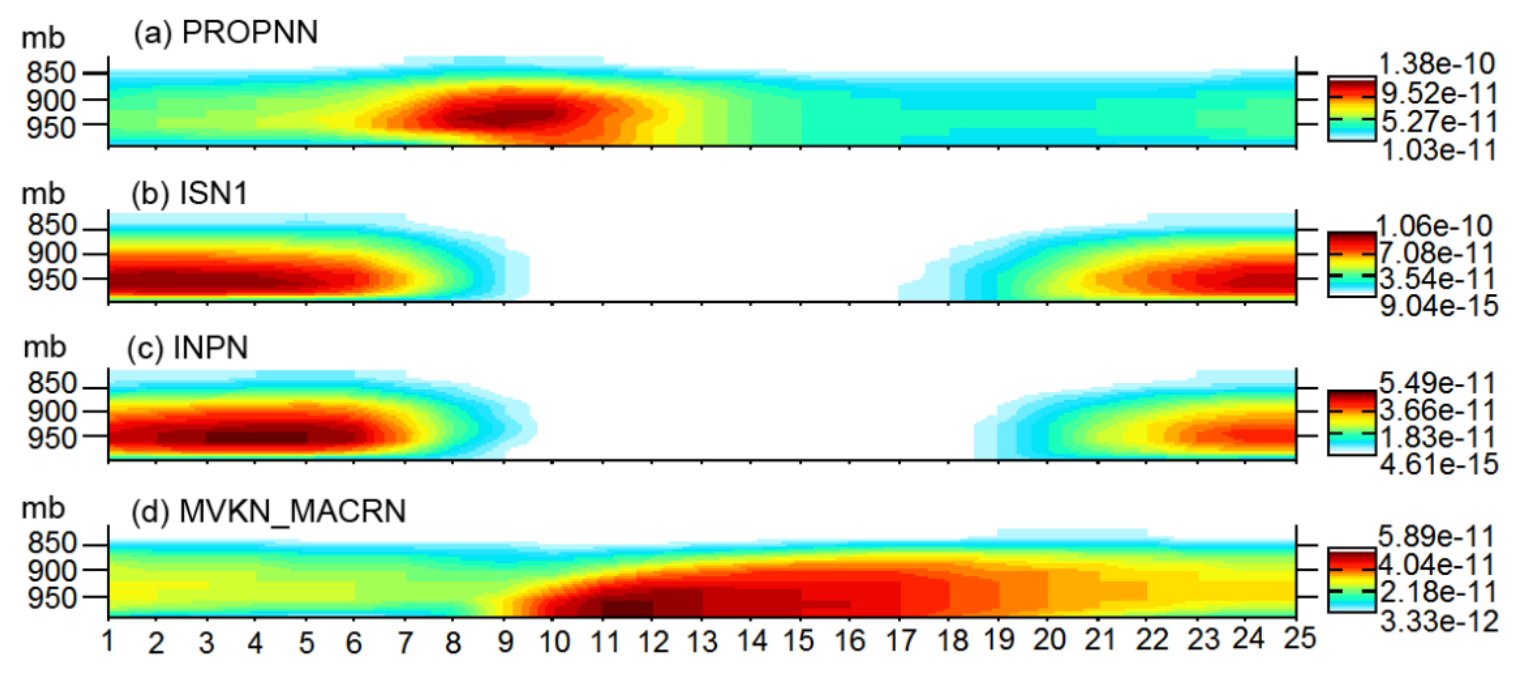

Figure S7. Modeled diurnal variation of PROPNN, ISN1, INPN and MVKN + MACRN (MVKN_MACRN) in the boundary layer of the Southeast U.S. The x-axis is the local time, and $y$-axis is the pressure. Color bar indicates the concentration in volume mixing ratio. 


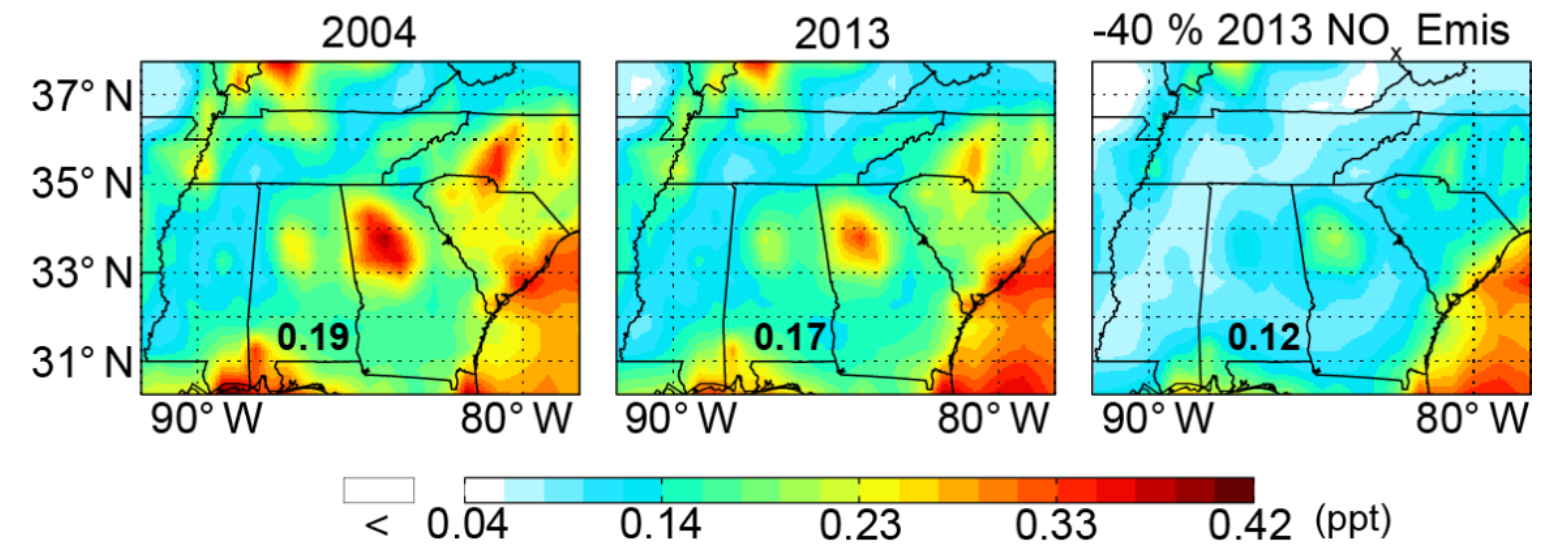

Figure S8. Averaged $\mathrm{OH}$ concentration from 10:00 to 14:00 LT during July-August of 2004, 2013, and a scenario with $40 \%$ reduction of anthropogenic $\mathrm{NO}_{\mathrm{x}}$ emissions of 2013 from AM3. Bold number in each panel is the regionally-averaged $\mathrm{OH}$ concentration. 


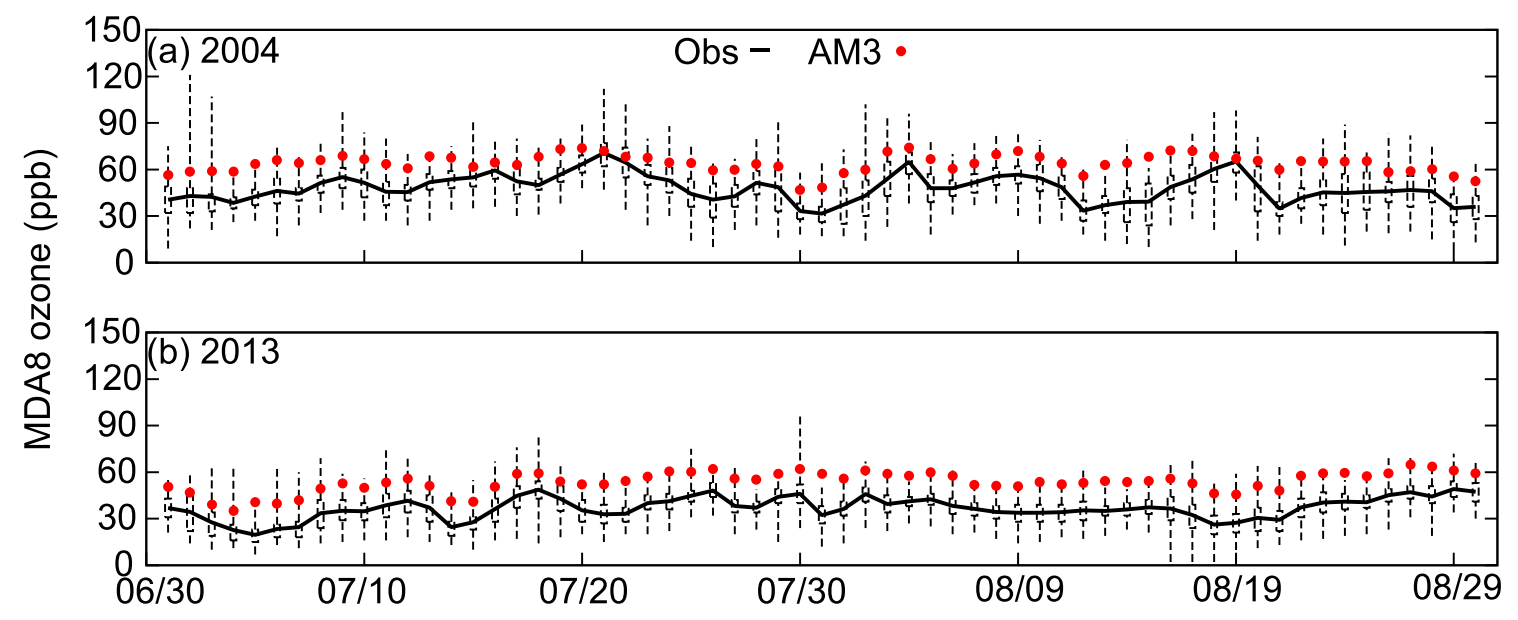

Figure S9. Daily MDA8 ozone during July-August of 2004 (a) and July-August of 2013 (b) averaged over 157 EPA AQS monitoring sites shown in Fig. S2. Horizontal axis is month and day of the year. Black lines, dash boxes and whiskers are the mean, interquartile range and lowest and highest observations; red dots are the mean of AM3. 


\section{References}

Atkinson, R., and Arey, J.: Gas-phase tropospheric chemistry of biogenic volatile organic compounds: a review, Atmos. Environ., 37, 197-219, 2003.

Fisher, J. A., Jacob, D. J., Travis, K. R., Kim, P. S., Marais, E. A., Miller, C. C., Yu, K., Zhu, L., Yantosca, R. M., and Sulprizio, M. P.: Organic nitrate chemistry and its implications for nitrogen budgets in an isoprene- and monoterpene-rich atmosphere: constraints from aircraft (SEAC4RS) and ground-based (SOAS) observations in the Southeast US, Atmos. Chem. Phys., 16, 1, 1-38, 2016.

Lee, L., Teng, A. P., Wennberg, P. O., Crounse, J. D., and Cohen, R. C.: On Rates and Mechanisms of $\mathrm{OH}$ and $\mathrm{O} 3$ Reactions with Isoprene-Derived Hydroxy Nitrates, J. Phys. Chem. A, 118, 9, 1622-1637, 2014.

Müller, J. F., Peeters, J., and Stavrakou, T.: Fast photolysis of carbonyl nitrates from isoprene, Atmos. Chem. Phys., 14, 5, 2497-2508, 2014.

Paulot, F., Crounse, J. D., Kjaergaard, H. G., Kroll, J. H., Seinfeld, J. H., and Wennberg, P. O.: Isoprene photooxidation: new insights into the production of acids and organic nitrates, Atmos. Chem. Phys., 9, 4, 1479-1501, 2009.

Pye, H. O. T., Luecken, D. J., Xu, L., Boyd, C. M., Ng, N. L., Baker, K. R., Ayres, B. R., Bash, J. O., Baumann, K., Carter, W. P. L., Edgerton, E., Fry, J. L., Hutzell, W. T., Schwede, D. B., and Shepson, P. B.: Modeling the Current and Future Roles of Particulate Organic Nitrates in the Southeastern United States, Environ. Sci. Technol., 49, 24, 14195-14203, 2015.

Saunders, S. M., Jenkin, M. E., Derwent, R. G., and Pilling, M. J.: Protocol for the development of the Master Chemical Mechanism, MCM v3 (Part A): tropospheric degradation of non-aromatic volatile organic compounds, Atmos. Chem. Phys., 3, 1, 161180, 2003.

Schwantes, R. H., Teng, A. P., Nguyen, T. B., Coggon, M. M., Crounse, J. D., St. Clair, J. M., Zhang, X., Schilling, K. A., Seinfeld, J. H., and Wennberg, P. O.: Isoprene NO3 Oxidation Products from the RO2 + HO2 Pathway, J. Phys. Chem. A, 119, 40, 1015810171, 2015.

Tyndall, G., Cox, R., Granier, C., Lesclaux, R., Moortgat, G., Pilling, M., Ravishankara, A., and Wallington, T.: Atmospheric chemistry of small organic peroxy radicals, J.

Geophys. Res., 106, D11, 12157-12182, 2001. 